\title{
Comparison between concurrent and sequential chemoradiation for non-small cell lung cancer in vitro
}

\author{
SEO-YOUNG SONG ${ }^{1}$, AMIT K. DAS ${ }^{2}$ and JOHN D. MINNA ${ }^{2}$ \\ ${ }^{1}$ Department of Internal Medicine, Kangwon National University Hospital, Chuncheon-si, Gangwon-do 200-722, \\ Republic of Korea; ${ }^{2}$ Hamon Cancer Center for Therapeutic Oncology and Simmons Cancer Center, \\ University of Texas Southwestern Medical Center, Dallas, TX 75390, USA
}

Received June 6, 2013; Accepted November 21, 2013

DOI: $10.3892 / \mathrm{ol} .2013 .1707$

\begin{abstract}
Current practice guidelines recommend the combination of chemotherapy and thoracic radiation for locally advanced non-small cell lung cancer (NSCLC). Previous meta-analyses have shown that concurrent chemoradiation (CCRT) may be superior to sequential chemoradiation (SCRT). However, few previous in vitro studies have analyzed these two treatment schedules. In the current study, four lung cancer cell lines harboring wild-type epidermal growth factor receptor, comprising two squamous and two non-squamous cell lines, were used. The $\mathrm{IC}_{10}$ concentrations of three platinum-based regimens were combined with radiation treatment. Cells were irradiated at 0, 2, 4, 6 and 8 Gy using a ${ }^{137} \mathrm{Cs}$ irradiator concurrently or sequentially. Surviving fractions (SFs) were plotted as a function of the radiation dose. In A549 cells, only the docetaxel (Doc) and carboplatin (Carbo) combination showed a significant radiosensitizing effect with CCRT treatment. For the other three cell lines, no difference was identified in the SFs between CCRT and SCRT. An in vitro method of comparing CCRT with SCRT was established using lung cancer cell lines. Overall, no significant difference was detected in the radiosensitizing effect of the two treatment schedules, with the exception of the A549 cell lines treated with Doc/Carbo.
\end{abstract}

\section{Introduction}

Lung cancer is the most common cause of cancer-related mortality worldwide (1). Non-small cell lung cancer (NSCLC) accounts for $80 \%$ of all types of lung cancer, and up to one-third of all patients with NSCLC present with

Correspondence to: Dr Seo-Young Song, Department of Internal Medicine, Kangwon National University Hospital, Kangwon National University School of Medicine, 17-1 Hyoja 3-dong, Chuncheon-si, Gangwon-do 200-947, Republic of Korea

E-mail: sysong@kangwon.ac.kr

Key words: chemotherapy, radiation, concurrent, sequential, carcinoma, non-small cell lung cancer, cell line locally advanced disease that is surgically unresectable. Current practice guidelines recommend that these cases be treated with a combination of chemotherapy and thoracic radiation (2). The two used methods of combining these two modalities are concurrent chemoradiation (CCRT), defined as chemotherapy administered on the same day as radiotherapy, and sequential chemoradiation (SCRT), usually administered as two to four cycles of chemotherapy prior to radiotherapy. The NSCLC Collaborative Group performed a meta-analysis of six randomized trials that compared the outcomes of SCRT versus CCRT (3). The chemotherapy regimens varied among the trials, but all incorporated a platinum-based agent. The meta-analysis showed that CCRT yielded improved local control and median survival rates, possibly due to its radiosensitizing effect. However, CCRT has been associated with more toxic adverse events, particularly treatment-related mortality and acute esophagitis. Furthermore, previous trials have exhibited poor patient accrual and have been terminated prematurely, therefore, the statistical power of these studies has been insufficient to detect a significant benefit. Thus, certain authors have insisted that future studies are required to support CCRT as the standard of care (4).

Compared with clinical data, little in vitro data concerning these two treatment schedules are available. The present study conducted in vitro chemoradiation using NSCLC cell lines to detect differences in chemoradiosensitivity according to the treatment schedule used.

\section{Materials and methods}

Cell culture. Four NSCLC cell lines, A549 (adenocarcinoma), H1299 (large cell neuroendocrine carcinoma), HCC15 [squamous cell carcinoma (SQCC)] and H157 (SQCC) were obtained from the Hamon Cancer Center for Therapeutic Oncology Research, University of Texas Southwestern Medical Center (Dallas, TX, USA). These cell lines were maintained at $37^{\circ} \mathrm{C}$ and $5 \% \mathrm{CO}_{2}$ in RPMI 1640 with glutamine (Hyclone, Logan, UT, USA) containing 5\% fetal bovine serum (FBS; Gemini Bio Products, West Sacramento, CA, USA).

Colony formation assay. The following three platinum-based regimens were used: Gemcitabine (Gem)/cisplatin (Cis), 
Table I. $\mathrm{IC}_{10}$ values of three platinum doublets for each cell line.

\begin{tabular}{|c|c|c|c|c|c|c|}
\hline \multirow[b]{2}{*}{ Cell line } & \multicolumn{2}{|c|}{$\mathrm{Gem} / \mathrm{Cis}, \mathrm{nM} / \mathrm{nM}$} & \multicolumn{2}{|c|}{$\mathrm{Pem} / \mathrm{Cis}, \mu \mathrm{m} / \mu \mathrm{m}$} & \multicolumn{2}{|c|}{ Doc/Carbo, nM/nM } \\
\hline & Gem & Cis & Pem & Cis & Doc & Carbo \\
\hline A549 & 0.486 & 0.034 & 0.412 & 0.123 & 0.127 & 1.476 \\
\hline H1299 & 0.170 & 0.119 & 0.040 & 0.012 & 0.038 & 0.443 \\
\hline HCC 15 & 0.922 & 0.065 & 0.126 & 0.028 & 0.030 & 0.346 \\
\hline H157 & 0.198 & 0.014 & 0.121 & 0.036 & 0.070 & 0.810 \\
\hline
\end{tabular}

Gem; gemcitabine; Cis, cisplatin; Pem, pemetrexed; Doc, docetaxel; Carbo, carboplatin.

Concurrent chemoradiation (CCRT)

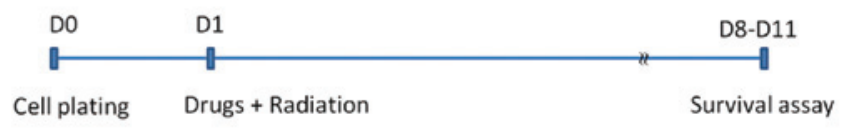

Sequential chemoradiation (SCRT)

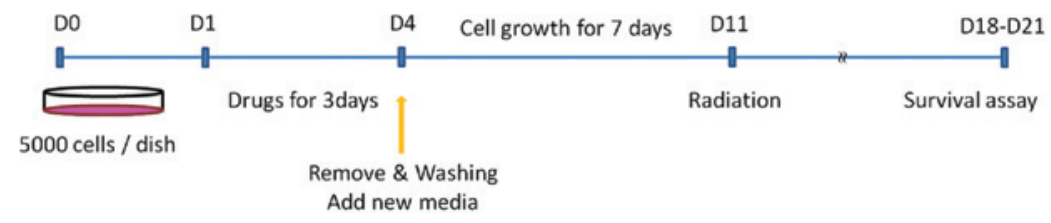

Figure 1. Treatment scheme for concurrent chemoradiation (CTRT) and SCRT in vitro. D, day.

pemetrexed (Pem)/Cis and docetaxel (Doc)/carboplatin (Carbo). For chemotherapy combined with radiation, the $\mathrm{IC}_{10}$ values of these three platinum-based regimens were determined using colony formation assays. The results are presented as the $\mathrm{IC}_{10}$ values of Gem, Pem and Doc in each combination. The $\mathrm{IC}_{10}$ values of $\mathrm{C}$ is and Carbo were then calculated using the drug ratio based on the doses used in the clinic.

Clonogenic cell survival assay. Fig. 1 shows the in vitro chemoradiation treatment scheme. For CCRT, the cells were seeded in triplicate six-well plates containing RPMI 1640 plus $10 \%$ FBS at various densities commensurate with the dose of radiation. These cells were treated with chemotherapy at the $\mathrm{IC}_{10}$ and with radiotherapy on the same day. For SCRT, the cells were plated on 100-mm dishes and treated with chemotherapy at the $\mathrm{IC}_{10}$ on day 1 (D1). To terminate drug exposure, the medium was removed and the dishes were then washed twice with medium on D4. The cells were allowed to grow for seven days and then irradiated on D11 following replating on six-well plates.

The cells were irradiated at various doses $(0,2,4$, 6 and 8 Gy) using a ${ }^{137}$ Cs irradiator (Mark I-68; J. L. Shepherd and Associates, San Fernando, CA, USA). Colonies containing $>50$ cells were stained with crystal violet and manually counted using a microscope. The percentage cell viability of the irradiated samples [shown as plating efficiency (PE)] was calculated as the fraction of cell viability relative to $100 \%$ viability of the untreated samples and plotted as a function of the radiation dose. Surviving fractions (SFs) were plotted as a function of the radiation dose. Cell survival curves were generated using the following multitarget, single-hit cell survival equation:

$$
S=1-\left(1-e^{-D / D_{0}}\right)^{n}
$$

$\mathrm{S}$ indicates the $\mathrm{SF}$ at dose $\mathrm{D}$, while $\mathrm{D}_{0}$ indicates the dose required to reduce the $\mathrm{SF}$ to $37 \%$ and $\mathrm{n}$ indicates the total number of targets at $0 \mathrm{~Gy}$. The radiation sensitivity was expressed as the SF at 2 Gy (SF2).

\section{Results}

Table I shows the $\mathrm{IC}_{10}$ values for each doublet. To these concentrations, radiation was then added concurrently or sequentially. In the H1299, HCC15 and H157 cells, no differences were identified between CCRT and SCRT (Fig. 2B-D). For the HCC15 cells, even if the PE of the control in the sequential arm was lower than others, no significant decrease was identified in SF2 value. In the A549 cells, only the Doc/Carbo combination showed a significant radiosensitizing effect in CCRT (Fig. 2A).

\section{Discussion}

In locally advanced NSCLC, combined-modality chemoradiation is usually used in current practice. CCRT is likely to be superior to SCRT according to the results of several previous studies $(2,5,6)$. However, CCRT is known to be more toxic in terms of esophagitis and pneumonitis. The effect of the 
A

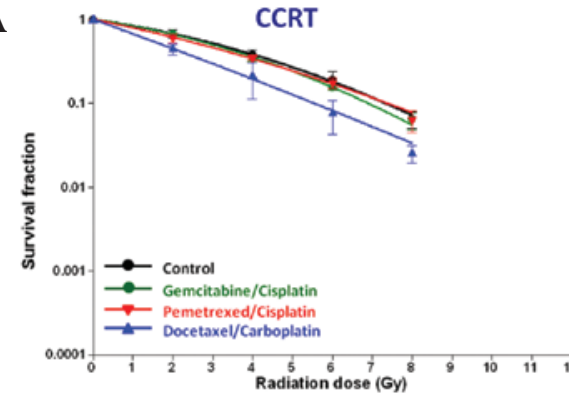

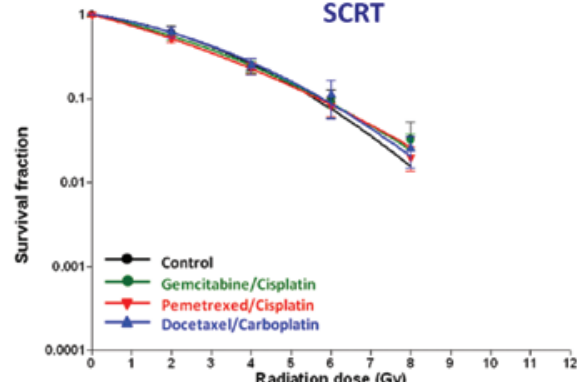

\begin{tabular}{|c|c|c|c|c|c|c|c|c|}
\hline \multirow[t]{2}{*}{ A549 } & \multicolumn{4}{|c|}{ Concurrent CRT } & \multicolumn{4}{|c|}{ Sequential CRT } \\
\hline & Control & Gem/Cis & Pem/Cis & Doc/Carbo & Control & Gem/Cis & Pem/Cis & Doc/Carbo \\
\hline PE (\%) & $69.1 \pm 10.3$ & $63.6 \pm 1.80$ & $62.5 \pm 11.2$ & $47.6 \pm 2.08$ & $68.5 \pm 10.7$ & $59.3 \pm 5.49$ & $63.0 \pm 6.50$ & $59.7 \pm 7.88$ \\
\hline SF2 & 0.675 & 0.666 & 0.6 & 0.438 & 0.577 & 0.545 & 0.517 & 0.574 \\
\hline$p$-value & & 0.755 & 0.057 & $<0.001$ & & 0.323 & 0.058 & 0.878 \\
\hline
\end{tabular}

B
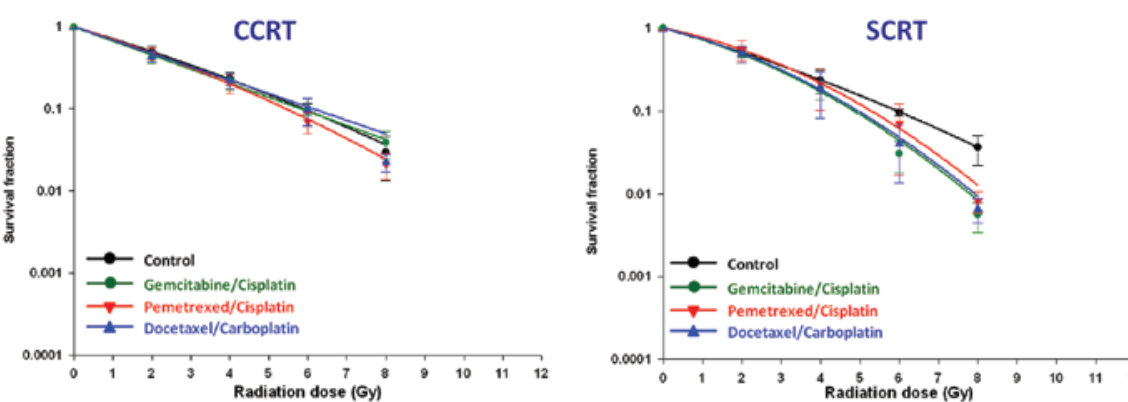

\begin{tabular}{|c|c|c|c|c|c|c|c|c|}
\hline \multirow[t]{2}{*}{ H1299 } & \multicolumn{4}{|c|}{ Concurrent CRT } & \multicolumn{4}{|c|}{ Sequential CRT } \\
\hline & Control & Gem/Cis & Pem/Cis & Doc/Carbo & Control & Gem/Cis & Pem/Cis & Doc/Carbo \\
\hline PE (\%) & $40.8 \pm 9.33$ & $33.4 \pm 5.17$ & $34.9 \pm 3.39$ & $34.1 \pm 4.50$ & $35.8 \pm 10.7$ & $31.4 \pm 12.5$ & $29.3 \pm 9.97$ & $29.8 \pm 8.60$ \\
\hline SF2 & 0.52 & 0.428 & 0.506 & 0.456 & 0.511 & 0.481 & 0.579 & 0.495 \\
\hline$p$-value & & 0.052 & 0.427 & 0.112 & & 0.273 & 0.459 & 0.666 \\
\hline
\end{tabular}

C
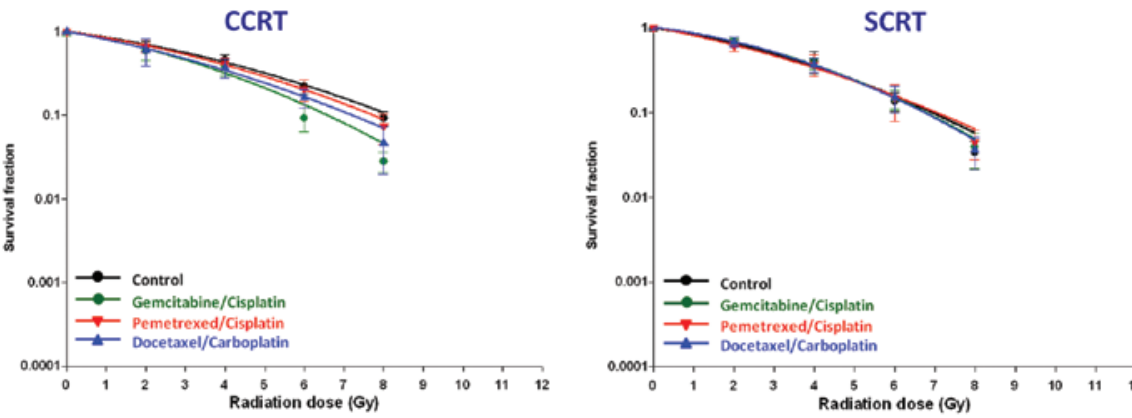

\begin{tabular}{|c|c|c|c|c|c|c|c|c|}
\hline \multirow[t]{2}{*}{ HCC15 } & \multicolumn{4}{|c|}{ Concurrent CRT } & \multicolumn{4}{|c|}{ Sequential CRT } \\
\hline & Control & Gem/Cis & Pem/Cis & Doc/Carbo & Control & Gem/Cis & Pem/Cis & Doc/Carbo \\
\hline PE (\%) & $57.2 \pm 19.1$ & $50.3 \pm 19.3$ & $52.6 \pm 16.6$ & $53.2 \pm 23.8$ & $27.1 \pm 8.52$ & $40.3 \pm 13.6$ & $44.3 \pm 16.7$ & $43.3 \pm 10.6$ \\
\hline SF2 & 0.674 & 0.587 & 0.668 & 0.604 & 0.62 & 0.671 & 0.599 & 0.677 \\
\hline$p$-value & & 0.164 & 0.904 & 0.417 & & 0.182 & 0.581 & 0.153 \\
\hline
\end{tabular}

$\mathbf{D}$
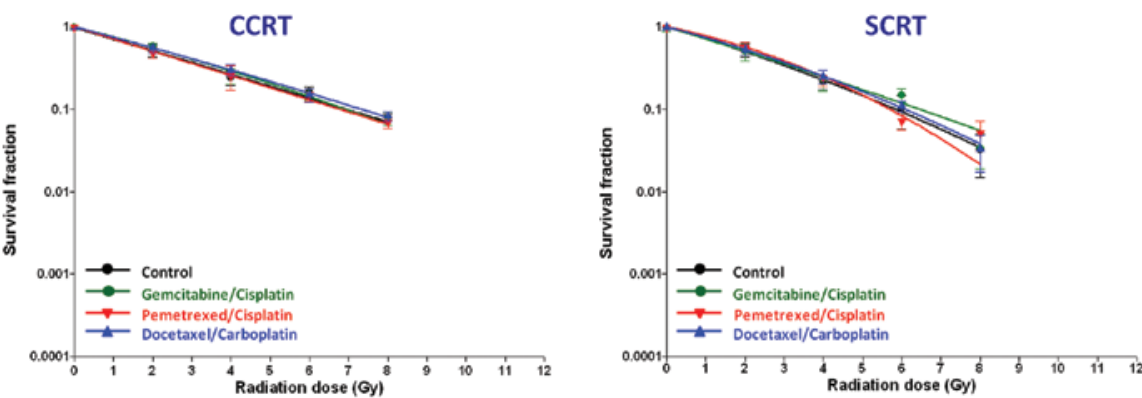

\begin{tabular}{|c|c|c|c|c|c|c|c|c|}
\hline \multirow[t]{2}{*}{ H157 } & \multicolumn{4}{|c|}{ Concurrent CRT } & \multicolumn{4}{|c|}{ Sequential CRT } \\
\hline & Control & Gem/Cis & Pem/Cis & Doc/Carbo & Control & Gem/Cis & Pem/Cis & Doc/Carbo \\
\hline $\operatorname{PE}(\%)$ & $50.1 \pm 12.3$ & $46.8 \pm 14.2$ & $45.1 \pm 12.3$ & $47.7 \pm 13.1$ & $42.0 \pm 19.2$ & $34.6 \pm 11.1$ & $40.5 \pm 14.7$ & $43.3 \pm 17.4$ \\
\hline SF2 & 0.511 & 0.578 & 0.48 & 0.556 & 0.505 & 0.509 & 0.573 & 0.535 \\
\hline$p$-value & & 0.012 & 0.331 & 0.126 & & 0.909 & 0.011 & 0.281 \\
\hline
\end{tabular}

Figure 2. Results of the chemoradiation for each cell line; (A) A549, (B) H1299, (C) HCC15 and (D) H157. CCRT, concurrent chemoradiation; SCRT, sequential chemoradiation; Gem; gemcitabine; Cis, cisplatin; Pem, pemetrexed; Doc, docetaxel; Carbo, carboplatin; PE, plating efficiency, SF2, surviving fraction at 2 Gy. 
radiation schedule in vitro is not known, since no data exists. The current study conducted chemoradiation concurrently or sequentially to determine the effects of the three platinum doublets in lung cancer cell lines. Platinum-based chemotherapy regimens are used widely, but to date, no consensus has been reached with regard to the optimal chemotherapeutic regimen. The present study assessed three doublets that are widely used in clinical practice. The cytotoxicity of an agent is usually expressed as the $\mathrm{IC}_{50}$ value. However, the $\mathrm{IC}_{10}$ was used since the majority of cells were unable to survive treatment with the $\mathrm{IC}_{50}$ concentration when combined with radiation.

The four cell lines used in the current study harbored wild-type epidermal growth factor receptor (EGFR). Das et al identified a subset of naturally occurring $E G F R$ mutations that lack a critical radioprotective function of EGFR (7). In the future, additional studies using more cell lines must compare the two treatment schedules according to mutational status. The methods used in the current study are likely to facilitate conducting such in vitro studies of combination modalities.

In conclusion, the present study established an in vitro method of comparing CCRT with SCRT using lung cancer cell lines. Overall, no significant difference was detected in the radiosensitizing effect of the two treatment schedules, with the exception of the A549 cell line treated with Doc/Carbo. The underlying mechanism should be elucidated.

\section{Acknowledgements}

The present study was partly supported by a grant from the National R\&D Program for Cancer Control, Ministry for Health and Welfare, Republic of Korea (no. 1020420) and the Kangwon National University.

\section{References}

1. Jemal A, Siegel R, Ward E, et al: Cancer statistics, CA Cancer J Clin 58: 71-96, 2008.

2. National Comprehensive Cancer Network: NCCN Guidelines for non-small cell lung cancer. http://www.nccn.org/professionals/physician_gls/pdf/nscl.pdf. Accessed April 2, 2013.

3. Aupérin A, Le Péchoux C, Rolland E, et al: Meta-analysis of concomitant versus sequential radiochemotherapy in locally advanced non-small-cell lung cancer. J Clin Oncol 28: 2181-2190, 2010.

4. O'Rourke N and Macbeth F: Is concurrent chemoradiation the standard of care for locally advanced non-small cell lung cancer? A review of guidelines and evidence. Clin Oncol (R Coll Radiol) 22: 347-355, 2010.

5. O'Rourke N, Roqué I Fuguls M, Farré Bernadó N and Macbeth F: Concurrent chemoradiotherapy in non-small cell lung cancer. Cochrane Database Syst Rev 6: CD002140, 2010.

6. El-Sharouni SY, Kal HB, Battermann JJ and Schramel FM: Sequential versus concurrent chemo-radiotherapy in inoperable stage III non-small cell lung cancer. Anticancer Res 26: 495-505, 2006.

7. Das AK, Chen BP, Story MD, et al: Somatic mutations in the tyrosine kinase domain of epidermal growth factor receptor (EGFR) abrogate EGFR-mediated radioprotection in non-small cell lung cancer. Cancer Res 67: 5267-5274, 2007. 